\title{
Evolution of proteomic biomarker for chronic liver disease: Promise into reality
}

Journal of Circulating Biomarkers

\author{
Krishna Sumanth Nallagangula', KN Shashidhar', \\ V Lakshmaiah ${ }^{2}$, and Muninarayana ${ }^{3}$
}

\begin{abstract}
Liver is the vital organ for synthesis of proteins whose concentration in blood reflects liver dysfunction. Variations in protein domain can generate clinically significant biomarkers. Biomarker pipeline includes discovery of candidates, qualification, verification, assay optimization, and validation. Advances in proteomic approach can discover protein biomarker candidates based on "up-or-down" regulation or fold change in expression which is correlated with disease state. Despite numerous biomarker candidates been discovered, only few are useful in clinical practice which indicates the need for well-established validation regimen. Hence, the main purpose of this review is to understand the protein biomarker development and pitfalls. Companion diagnostics provide insights into potential cost-effective diagnosis for chronic liver disease.
\end{abstract}

\section{Keywords}

Chronic liver disease, proteomics, biomarker discovery, assay optimization, specificity and sensitivity, validation strategies, pitfalls, companion diagnostics

Date received: 22 December 2017; accepted: 18 April 2018

\section{Introduction}

Human liver is a vital organ for health and survival, performing biochemical functions, namely, protein synthesis, production of digestive enzymes, and detoxification. ${ }^{1}$ Liver fibrosis/cirrhosis is a pathological condition in which functions of liver are impaired by chronic liver insult. Cirrhosis of liver is the histological development of regenerative nodules surrounded by fibrous bands in response to chronic liver injury which leads to portal hypertension and endstage liver disease. Fibrosis is a reversible natural wound healing response to chronic liver injury resulting in accumulation of extra cellular matrix (ECM); precursor of cirrhosis. Despite varied etiology, the pathological characteristics which include degeneration, necrosis of hepatocytes, and replacement of liver parenchyma by fibrotic tissues and regenerative nodules are common and ultimately result in liver dysfunction. ${ }^{2}$

After an acute injury, there will be regeneration and replacement of liver parenchymal cells to necrotic and apoptotic cells. If injury persists, there will be substitution of hepatocytes with abundant ECM having contractile, inflammatory, and fibrogenic properties. ${ }^{3}$ Activation of hepatic stellate cells (HSCs) is a crucial step of tissue injury and regeneration. ${ }^{4}$ Quiescent HSCs present in space of Disse will be activated and trans-differentiate into myofibroblasts like cells which are responsible for ECM production and accumulation in injured liver. ${ }^{5}$ Accumulation of

\footnotetext{
'Department of Biochemistry, Sri Devaraj Urs Medical College, SDUAHER, Karnataka, India

${ }^{2}$ Department of Medicine, Sri Devaraj Urs Medical College, SDUAHER, Karnataka, India

${ }^{3}$ Department of Community Medicine, Sri Devaraj Urs Medical College, SDUAHER, Karnataka, India

\section{Corresponding Author:}

KN Shashidhar, Department of Biochemistry, Sri Devaraj Urs Medical College, Kolar, Karnataka 563103, India.

Email: drshashikn197।@yahoo.co.in
} 


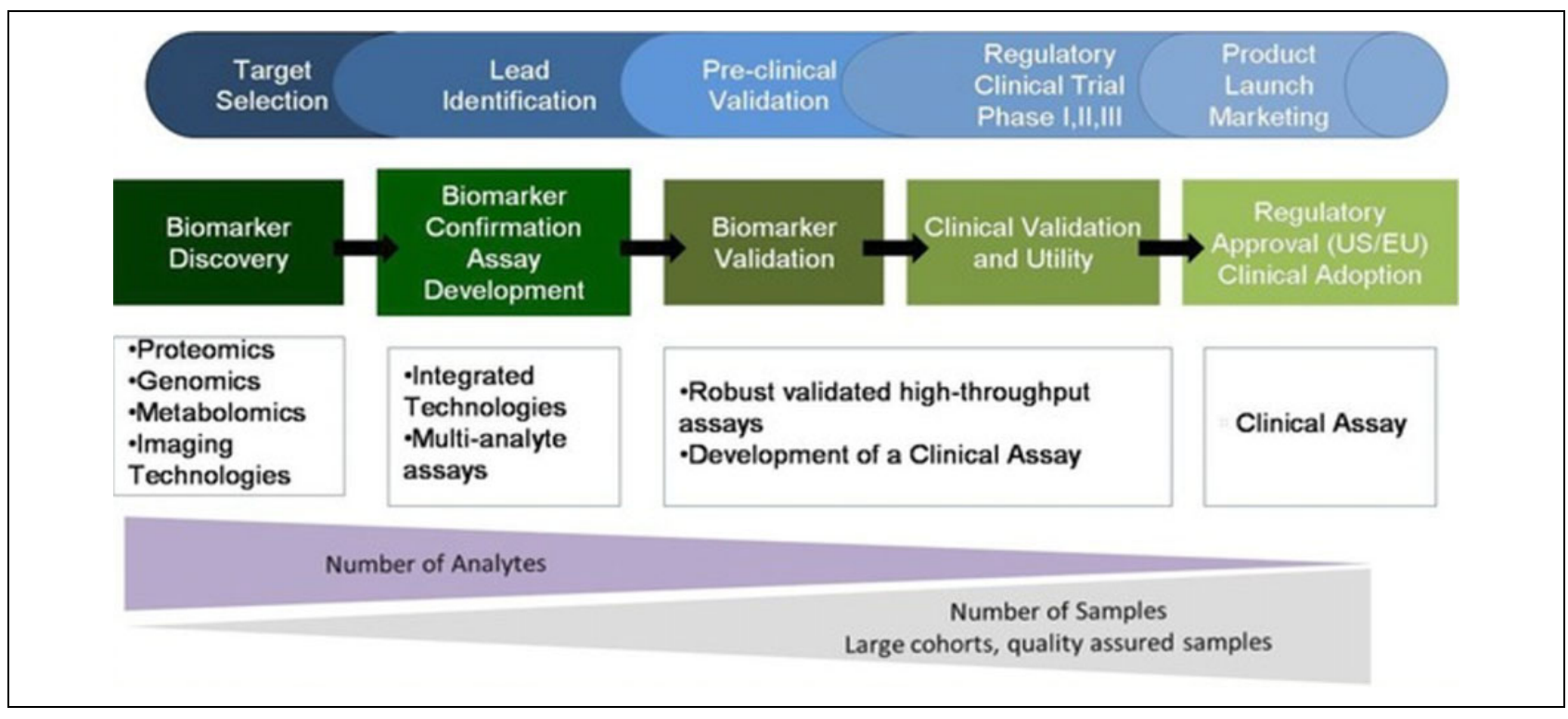

Figure I. Biomarker discovery and validation; advances in omics will generate clinically significant biomarker candidates which need validation through verification studies and clinical assay development. Source: Adapted from the study by Nies et al. ${ }^{12}$

ECM is due to increased synthesis and decreased degradation by over expression of tissue inhibitors of metallo proteinases which inhibits matrix metallo proteinases. Fibrotic liver contains 3-10 times more ECM compared to normal liver which includes collagen types, glycoproteins, proteoglycans, and glycosaminoglycans. ${ }^{2}$

Accurate assessment of degree of hepatotoxicity especially at early stage is crucial for clinical management to predict prognosis and therapeutic decision even to reverse liver fibrosis/cirrhosis to normal architecture of liver. Despite development of potential diagnostic tests, for the past 50 years, liver biopsy is still considered as gold standard for diagnosis of chronic liver diseases (CLDs), which is associated with pain and complications. Biomarkers are used to diagnose or monitor the activity of disease and to assess therapeutic response for CLD. ${ }^{2}$

A biomarker is a molecule that is analytically measured with well-established performance characteristics in an established scientific frame work of evidence that elucidates physiological, toxicological, pharmacological, or clinical outcome. ${ }^{6}$ Biomarkers can be gene variants, single nucleotide polymorphisms, gene expression products, metabolites, polysaccharides, circulating nucleotides, and proteins. ${ }^{7}$

Validation of a biomarker includes assessing the biomarker, measuring the performance characteristics, and determining the range of conditions for reproducibility and accuracy. Biomarker validation relates biomarker with biological process and clinical end point and is necessary for fit-for-purpose. Validation helps research data for better patient care. An ideal biomarker for CLD should be specific, sensitive to indicate active damage, prior to histological changes, easily accessible in peripheral tissue, and cost-effective. ${ }^{2,6}$ A biological marker objectively measures and evaluates normal biological, pathogenic process, or pharmacological response to a drug. Surrogate markers serve as a substitute for a clinically meaningful end point. Prognostic biomarker indicates likely outcome of a disease irrespective of treatment. Predictive biomarker helps to assess response to a particular treatment. Pharmacodynamic biomarker gives interaction between drug and target. $^{6,7}$ In this review, an attempt has been made to understand the process of proteomic biomarker development which includes protein biomarker discovery, validation, and pitfalls in biomarker pipeline for CLD.

\section{Biomarker development by proteomic approach}

Establishment of correlation between disease state and biomarker alterations will help clinician for diagnosis and tailored therapy. ${ }^{8}$ In CLD, protein domain will have alterations where the amount of protein from liver enters into circulation and serves as an indicator for degree of liver dysfunction, which holds good for discovery of novel protein biomarkers using proteomics. ${ }^{9}$ Proteins have more structural diversity and stability than DNA and RNA and carry more information than nucleic acids which are dynamic and reflection of cellular physiology. ${ }^{10}$ Advances in proteomic approach help discover and identify clinically significant protein biomarker candidates for CLD. Protein biomarker pipeline includes a series of essential components such as discovery, research assay optimization, analytical and clinical validation, and clinical utility (Figure 1). ${ }^{11,12}$

\section{Protein biomarker candidate discovery}

Protein biomarker discovery is a simplified, semiquantitative, unbiased binary comparison between diseased and 


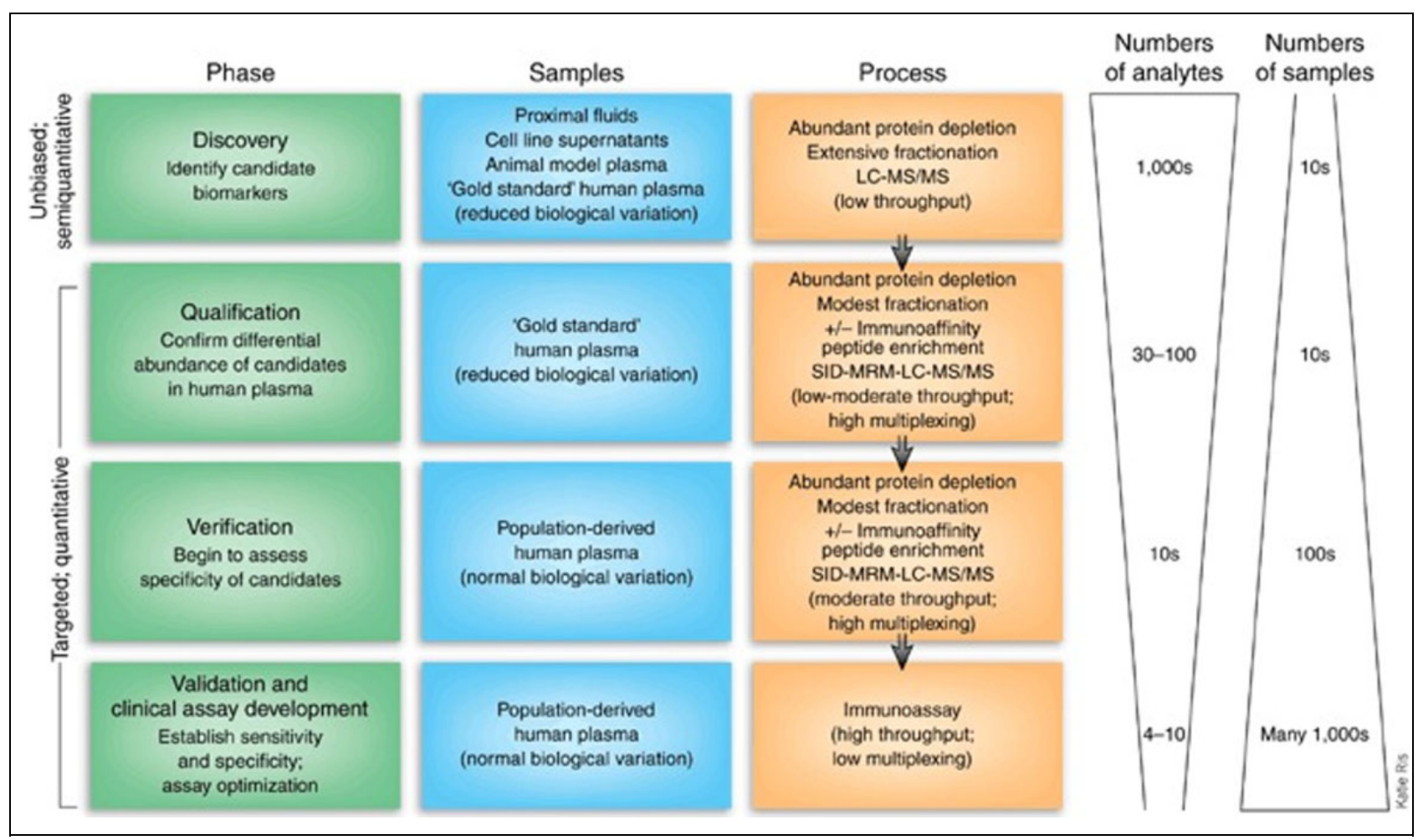

Figure 2. Discovery of protein biomarker; protocol for the development of protein biomarker candidate. Source: Adapted from the study by Rifai et al. ${ }^{13}$

normal using biological samples for maximal detection of significant protein expression differences. It needs avoiding contamination of other diseases and confounding factors. ${ }^{2,13}$ Proteins that are differentially expressed between CLD and normal are due to changes in translation, posttranslational modifications, and degradation or that are involved in pathophysiological changes which are good sources of biomarker candidates. ${ }^{10}$ Comparative analysis between diseased and healthy generates hundreds of protein biomarker candidates that are differentially expressed. There is an inverse relationship between number of samples analyzed and number of proteins quantified (Figure 2). ${ }^{13,14}$

Discovery of protein biomarker candidates for CLD, plasma/serum is the best choice among other body fluids, represents physiological and pathological process. ${ }^{15}$ During discovery phase, the variables (study design, preanalytical, and analytical) which affect precision should be minimized. Study population should be selected from a well-defined study design with definite inclusion and exclusion criteria to minimize bias. Case control study or cohort study usually considered as a better study design for discovery phase of biomarker. Multiple sources of bias could be seen in retrospective and observational studies. ${ }^{16}$ Preanalytical variables such as type and manufacture of collection tubes, phlebotomy device, patient's posture, time of sample collection, type of sample to be collected, storage conditions, and sample preparation should be controlled in order to get significant observation. Analytical variables such as mass resolution and collision energy need to be controlled to minimize the source of variations. ${ }^{13,14}$

In candidate discovery for CLD, to obtain significant protein expressional difference, use of gold standard sample is recommended. Plasma is the biofluid of choice (Human Proteomic Organization), and contains proteins that reflect a variety of human diseases. ${ }^{17}$ Anticoagulants (EDTA or citrate) cause osmotic shifting of fluid from cell to plasma, which gives $10 \%$ less values when compared to serum and are known to chelate cations, and give negative results in case protein of interest has cations in its structure. Antigenic epitope mask might happen which reduces immunoreactivity because of heparin. ${ }^{13,18}$ Compared to plasma, proteins are more stable in serum. For large studies, serum is the preferred sample by clinicians since it is the most simple matrix. ${ }^{14}$ Although individual sample analysis is recommended, pooling strategy with definite criteria from multiple individuals reduces sample number and cost. ${ }^{19}$

Protein biomarker discovery in plasma/serum is complicated. Around $99 \%$ of protein content is comprised of 20 abundant proteins which interfere in identification and characterization of low abundant proteins by mass spectral and electrophoretic analysis. ${ }^{20-23}$ Depletion of abundant proteins allows detection of low abundant proteins. But there is a chance for further removal of low abundant proteins that are bound to high abundant proteins. ${ }^{14}$ For 
accurate protein biomarker candidate discovery for CLD, depletion of albumin (55\% of total protein) and immunoglobulins may be achieved using high affinity columns. ${ }^{21,22}$ In two-dimensional gel electrophoresis (2-DE), depletion dilemma can be rectified using narrow $\mathrm{pH}(3-5.6)$ range which avoids interference of abundant proteins (albumin, transferrin, and immunoglobulins). ${ }^{9,14}$

After depletion, discovery may be carried out by fractionation and purification using different analytical methods: 2-DE for separation of proteins followed by identification of significant protein spots using software tools. Identified spots are subjected for in-gel digestion to identify peptides and proteins either by surfaceenhanced laser desorption-ionization (SELDI) or matrix-assisted laser desorption-ionization (MALDI) or liquid chromatography-mass spectrometry (LC-MS) and proteomics search engines, that is, Mascot or SEQUEST. ${ }^{24-26}$ Unlike gel-based discovery, LC-MS carried out before or after enrichment of proteins by trypsin digestion, splitting long proteins into short peptides followed by chromatographic separation in addition to mass to charge ratio. ${ }^{14,27,28}$

2-DE has limited sensitivity and reproducibility compared to LC-MS. The main disadvantage of SELDI/ MALDI is difficulty in detection of differential pattern and identification of peaks. Automated LC-MS is suitable for protein biomarker discovery. Secondary ions collected from chromatographic profiles from MS spectra are subjected to proteomic search engines. ${ }^{29,30}$ Identified peptides are used to determine differential expression between CLD and normal. Use of parametric statistical tools prior to peptide identification is recommended. Biomarker candidates reported and identified for CLD by one group of researchers are not identified by another group (Table 1) because of lack of standardization of multistep procedures. Selection of specific criteria during LC-MS gives complexity and errors for reproducibility between laboratories. Biomarker discovery and validation should be performed in a blinded fashion, free from bias, and performed in a similar fashion that remove all confounding factors and generate significant biomarker candidates. ${ }^{30}$

\section{Biomarker validation}

Biomarker validation is necessary to deliver high-quality research data for effective use of biomarker for better patient care. Great interest and technological advancement in biomarker discovery results in identification of protein biomarker candidates for CLD. Biomarker candidates require verification that demonstrates the differential expression which remains detectable by assay to be used for validation. ${ }^{13}$ Despite numerous biomarker candidates identification, verification may be done only for few qualified candidates in terms of marker performance and reagent availability. ${ }^{10}$ Proteins that act in cellular pathways and deregulated in CLD should be considered for further validation. ${ }^{19}$ Validation of biomarker and clinical assay optimization requires measurement of thousands of patient samples with narrow measurement coefficient of variation values. $^{13}$

\section{Assay optimization}

As MS is unable to achieve high measurement accuracy and precision, it is necessary to develop antibodies for quantification of biomarker candidates. Concentration of protein in serum or plasma ranges from picograms to nanograms per milliliter; highly sensitive immunotechniques are required for quantification. Enzyme-linked immunosorbent assay (ELISA) is the best alternative for quantification of these proteins compared to sophisticated nonimmunebased techniques. ${ }^{35}$ Capture and detection antibodies (monoclonal or polyclonal), which detect distinct epitope of the protein, are needed to form sandwich reaction. Specificity of antibodies is established using Western blot or immunostaining. During development of ELISA, care should be taken to minimize the effect of variables such as avidity, concentration of antibodies (monoclonal capture/detection $0.5-4 / 0.25-2 \mu \mathrm{g} / \mathrm{ml}$ and polyclonal capture/ detection $0.2-0.8 / 0.05-0.4 \mu \mathrm{g} / \mathrm{ml}$ ), incubation time and temperature, sample volume, dilution of sample, $\mathrm{pH}$, composition and concentration of diluents, enzyme, substrate, and quality of detector which affect performance characteristics. Fluorescent or chemiluminescent are other alternatives for better sensitivity. ${ }^{13,36}$

\section{Analytical evaluation}

Newly developed assay requires analytical validation before evaluating clinical utility in terms of performance characteristics such as outcome studies, clinical requirement, proficiency testing, and goals set by regulatory agencies. ${ }^{35}$ Preanalytical variables should be characterized and controlled in various physiological and pathological conditions. Time of collection of sample (fasting or fed state) should be defined. In fed state, chylomicrons do not affect ELISA. Selection of appropriate sample (plasma or serum) and use of anticoagulants should be determined. Storage conditions and duration of storage should be examined. Physiological factors such as age, gender, and ethnicity significantly affect protein concentrations along with lifestyle factors. Pathological conditions and drugs which influence protein concentrations should be examined before estimation. ${ }^{13}$

Indicators of accuracy, precision, analytical measurement range, and reference intervals should be defined. Trueness is the closeness of agreement between average measured values of different samples which reflect bias (systemic error). Accuracy is the closeness of agreement between the values measured and true concentration of analyte. ${ }^{37}$ Newly discovered methods usually do not have reference materials and methods and should use alternative 
Table I. Protein biomarker candidates identified by proteomic analysis for liver fibrosis.

\begin{tabular}{|c|c|c|c|c|}
\hline Authors & $\begin{array}{l}\text { Etiology of } \\
\text { liver fibrosis }\end{array}$ & Type of sample & Proteomic techniques & Protein biomarker candidates identified \\
\hline White et al. ${ }^{3 l}$ & $\mathrm{HCV}$ & Serum & 2-DE and LC-MS & $\begin{array}{l}\alpha 2 \text { macroglobulin } \\
\text { Haptoglobin } \\
\text { Complement } \mathrm{C} 4 \\
\text { Serum retinol binding protein } \\
\text { Apolipoprotein } \mathrm{Al} \\
\text { Apolipoprotein A-IV }\end{array}$ \\
\hline Gangadharan et al. ${ }^{32}$ & $\mathrm{HCV}$ & Serum & 2-DE and LC-MS & $\begin{array}{l}\alpha 2 \text { macroglobulin } \\
\text { Inter- } \alpha \text {-trypsin inhibitor heavy chain } \mathrm{H} 4 \\
\alpha \mathrm{I} \text { antichymotrypsin } \\
\text { Apolipoprotein LI } \\
\text { Paraoxonase/aryleserase I } \\
\text { Zinc- } \alpha 2 \text {-glycoprotein } \\
\text { CD5 antigen-like protein } \\
\beta 2 \text { glycoprotein I }\end{array}$ \\
\hline Gangadharan et al. ${ }^{9}$ & $\mathrm{HCV}$ & Serum & $\begin{array}{l}\text { 2-DE, LC-MS, and in-solution } \\
\text { isoelectric focusing }\end{array}$ & Beta chains of $\mathrm{C} 3$ and $\mathrm{C} 4$ \\
\hline Gangadharan et al. ${ }^{33}$ & $\mathrm{HCV}$ & Serum & 2-DE and LC-MS & $\begin{array}{l}\text { Adiponectin } \\
\text { Sex hormone binding protein } \\
\text { I4-3-3 protein zeta/delta } \\
\text { Complement C3dg } \\
\text { Immunoglobulin J chain } \\
\text { Apolipoprotein CIII } \\
\text { Corticosteroid binding globulin } \\
\alpha 2 \text { HS glycoprotein } \\
\text { Lipid transfer inhibitor protein } \\
\text { Haptoglobin-related protein }\end{array}$ \\
\hline Katrinli et al. ${ }^{34}$ & HBV & Liver tissue & 2-DE and LC-MS & $\begin{array}{l}\text { Apolipoprotein AI } \\
\text { Pyruvate kinase } \\
\text { Glyceraldehyde 3-phosphate dehydrogenase } \\
\text { Glutamate dehydrogenase } \\
\text { Alcohol dehydrogenase } \\
\text { Transferrin, peroxiredoxin } 3 \\
\text { Keratin 5, annexin }\end{array}$ \\
\hline $\begin{array}{l}\text { Nallagangula et al. (20|7) } \\
\text { (unpublished data) }\end{array}$ & ALD & Serum & 2-DE and LC-MS & $\begin{array}{l}\text { Serotransferrin } \\
\text { Keratin isoforms } \\
\text { Vitamin D binding protein isoform } 3 \\
\text { Angiotensinogen preproprotein } \\
\text { CD5 antigen-like protein } \\
\text { Hemopexin precursor } \\
\text { al antichymotrypsin } \\
\text { Glycerol kinase isoform XI } \\
\text { Sex hormone binding protein }\end{array}$ \\
\hline
\end{tabular}

HCV: hepatitis C virus; HBV: hepatitis B virus; ALD: alcoholic liver disease; 2-DE: two-dimensional electrophoresis; LC-MS: liquid chromatography-mass spectrometry.

protocols such as spike, recovery, and linearity. Use of specific antibodies should be necessary to have no cross reactivity with other proteins. Care should be taken during ELISA development to minimize the errors because of exogenous and endogenous substances. The factors (buffer components, sample matrix, compliment, and rheumatoid factor) can impact antibody binding in natural samples and therefore influence the accuracy of results should be ruled out. ${ }^{38}$ Repeatability is the measurement performed in the same condition, and reproducibility is the measurement performed in different conditions. To assess precision, two replicates per sample per run, and two runs per day for least 20 days are recommended.$^{39}$ Reference intervals must be defined for protein of interest and new methodology by comparing healthy individual values similar to those of patient values. ${ }^{39}$ Reference values should be subdivided into groups based on age, gender, race, and physiological states. Normal distribution of reference intervals for protein of interest for parametric analysis is presented as mean \pm 2SD and for nonparametric analysis will be presented as percentiles. Limits of detection and quantification must be defined with acceptable accuracy and precision. Limit of 
detection is the lowest value that exceeds the measurand value against blank sample which does not have protein of interest. Linearity gives the relation between observed value and expected value which is above the range of measurand values. ${ }^{13,39}$

\section{Clinical validation}

After analytical validation of new methodology for protein of interest, biomarker candidate should confirm the performance characteristics in terms of consistency and accuracy in clinical evaluation to diagnose or predict the clinical outcome of CLD. The newly identified biomarker candidate should satisfy the following criteria. ${ }^{40}$

\section{Sensitivity of biomarker}

The ability of a biomarker or change in magnitude of a biomarker with precision which is sensitive enough to reflect a meaningful change in clinical end point of CLD.

\section{Specificity of biomarker}

The ability of a biomarker or change in magnitude of a biomarker which distinguish patients who are responders and nonresponders in terms of change in clinical end point of CLD.

\section{Probability of false positive}

Desired change of biomarker is not reflected by positive change in clinical end point or even worse is associated with negative change in a clinical end point of CLD.

\section{Probability of false negative}

No change or small change is observed in magnitude of biomarker which fails to signal positive and meaningful change in a clinical end point of CLD.

\section{Pharmacokinetic/pharmacodynamic model}

Correlation between changes in biomarker and drug exposure, to predict future outcome or standardization of dose adjustments based on biomarker measurements.

Likelihood ratio of biomarker indicates certainty of the diagnosis of disease prevalence and calculates posttest odds of having a disease as the prevalence changes. Receiver operating characteristic (ROC) curve is the comparison of diagnostic accuracy of two or more tests and to define appropriate cutoff for clinical utility of test. Likelihood ratio and ROC curve are derived from sensitivity and specificity values. ${ }^{2,41}$

\section{Clinical utility}

Clinical utility predicts positive outcome of drug in selected and unselected groups. Novel biomarker candidate needs to be evaluated in a series of human population (sub and stratified). In phase I (exploratory phase), test results should be different from patients with confirmed CLD and those of control population without CLD. Area under ROC curve should be $>0.5$ for newly identified biomarker candidate to proceed further. In phase II (challenge phase), different cutoff values for sensitivity and specificity should be defined with diagnostic accuracy to predict the presence or the absence of CLD. Phase III (advanced clinical phase) is to establish diagnostic accuracy of biomarker in target population in different geographical regions independently. Phase IV (outcome phase) gives the positive influence of test to get healthy outcome of CLD by evaluating both tested and untested patients with respect to diagnostic and therapeutic intervention. ${ }^{13,42,43}$

\section{Pitfalls and limitations}

In current medical research, novel biomarker should have the ability to improve treatment which is cost-effective. ${ }^{7}$ Newly identified biomarkers for CLD are unable to replace the existing conventional markers in clinical practice due to errors in study design or experimental execution. Despite numerous biomarker candidates identified for CLD, few biomarkers only validated successfully. Pitfalls in biomarker pipeline are because of no proper relation between discovery, verification, and clinical validation (due to lack of definite selection criteria in discovery phase, biomarker verification (sensitivity and specificity), and less robustness in analytical validation) as well as less structure and scientific factors which fail to give strong evidence for better patient care (Figure 3). ${ }^{7,19}$

During discovery phase, appropriate and well characterized clinical specimen has impact on outcome of identification process. ${ }^{44}$ Selection of patients for biomarker studies should be done by specialist to ensure the presence or absence of the disease. Randomization and optimal selection of patients with single etiology are necessary and are well matched with age and gender with the same ethnicity. Other lifestyle factors play an important role in selection criteria, namely, body mass index, habits, physical activity as well as metabolic syndromes, and use of drugs. ${ }^{7}$ Small sample size and lack of information about history may give false negative values in discovery phase. ${ }^{13,45}$ Proper procedures for samples collection, handling, and storage to avoid denaturation of proteins should be followed. Systematic monitoring of quality of sample over a time period is necessary. ${ }^{7}$ Suitable semiquantitative methods and sophisticated technologies like LC-MS along with proper analysis and data interpretation can improve biomarker candidate quality and yield.

Validation of biomarker is expensive and timeconsuming. Protein biomarker quantification is essential to have specific detection and capture antibodies with high sensitivity to form sandwich method with low concentrated protein in biological specimens. Analysis of protein of 


\begin{tabular}{|ll|}
\multicolumn{2}{|c|}{ Pitfalls in Biomarker development } \\
$\begin{array}{l}\downarrow \\
\text { Discovery }\end{array}$ & \\
Lack in study design & Vailure in assay optimization \\
Personal and scientific failure & Less sensitivity \\
Funding issues & Less specificity \\
Small sample size & ROC $\leq 75 \%$ \\
No history of sample & Likelihood $\leq 5$ \\
No proper age and gender match & Odd ratio $\leq 1$ \\
Preanalytical & \\
Analytical & \\
Post analytical & \\
\hline
\end{tabular}

Figure 3. Pitfalls and limitations in biomarker development.

interest should be carried in triplicates and reported in mean and standard deviation. ${ }^{7,13}$ In research laboratory, quality control procedures are less compared to clinical laboratories, and basic steps should be implemented to get accuracy and precision. ${ }^{46}$ Difficulties in validation strategies, which need well-defined sub and stratified population matched with pathological and physiological factors of early disease state, are main reasons for pitfalls. Lack of sensitivity, specificity of biomarker for disease progression and regression with ROC $\leq 75 \%$, and likelihood ratio $\leq 5$ with odd ratio $\leq 1$ are limitations in biomarker pipeline. ${ }^{7}$

\section{Transition from research to routine}

Newly identified biomarker needs to satisfy and fulfill the need of application which can be able to separate patients into groups that clinicians would treat differently and could be able to give reliable outcome of the treatment and it should be evidence based. ${ }^{46}$ New test should add or replace the information provided by existing biomarkers for CLD and cost-effective for better patient care. ${ }^{47}$ Introduction of new biomarker from research laboratory into clinical laboratory is three-way collaboration involving research laboratory, diagnostic industry, and clinical laboratory. ${ }^{48}$ Care should be taken in research laboratory about selection of novel biomarker evaluation at early stage to minimize the methodological bias (preanalytical, analytical, and postanalytical) which may affect results. ${ }^{49,50}$ Validated biomarker in research laboratory will be transferred to specialist referral laboratory to confirm the assessment in clinical setting. ${ }^{47}$

Once new test has beneficial effect on patient outcome which is evidence based and cost-effective, it will be introduced into funded health care system. Biomarker has to meet analytical validation, quality control, external validation, personal qualification, training, and documentation for approval $(510(\mathrm{k}) /$ premarket approval (PMA)/in vitro diagnostic (IVD) Directive 98/97/EC) ${ }^{13,47}$ The test developed should be suitable for clinical laboratory and capable to meet basic requirements (robustness, stability of reagents, acceptable turnaround time, adaptability for automation, and low cost). If biomarker is ideally measured in serum which does not require special handling, storage with robust analytical procedure, rejection of samples will be minimized which decreases turnaround time. Internal quality control should be robust as external quality control may not be available for newly discovered and validated biomarkers. Interlaboratory comparison can provide information about accuracy and precision at early stages. Definite reference intervals and linearity range will help for best practice of biomarker. ${ }^{47}$

\section{Regulatory requirements}

IVD device to enter into market must meet the rules and regulations of $510(\mathrm{k})$ premarketing clearance or PMA by the food and drug administration (FDA) in the United States, the Pharmaceutical Affairs Law (PAL) and Market Authorization Holder by Pharmaceutical and Medical Devices Agency of Japan, and IVD Directive 98/97/EC by member states of European Union. According to FDA, $510(\mathrm{k})$ process is that new test should measure existing FDA classified analyte I or II where there is predicate cleared test which are commercially available. Information about new test should include classification, performance characteristics, and analytical capability (accuracy, precision, linearity, specificity, and sensitivity) comparison with that of existing predicate test. Class III, which is associated with high risk or clinical utility of biomarker or novel technological measurements or no predicate device, needs PMA process. ${ }^{13,51,52}$

\section{Companion diagnostics}

Companion diagnostics (CDx) is the central part of personalized medicine. CDx is simultaneous development of drug and diagnostic test: in vitro diagnostic device which provides information about safe and effective use of corresponding therapeutic product. CDx includes screening and detection, prognosis, monitoring, and theranostics. The key indicator for $\mathrm{CDx}$ is robustness of financing environment for drug and diagnostic companies which minimizes costs from selection of patient population till clinical trials. CDx improves chances for approval and increases market uptake. There is a need for CDx which can be able to provide diagnostic test specific for therapeutic drug for cost-effective and successful management of CLD., ${ }^{7,53}$

\section{Conclusion}

Early diagnosis of CLD is essential for disease management and even reversibility of liver fibrosis/cirrhosis. Concentration of proteins expressed from liver into circulation serves as an indicator for liver dysfunction and good source of biomarker development based on proteomic approach. Technological advancement generates biomarker candidates which is a prerequisite for validation in terms 
of performance characteristics, analytical validation, accuracy, precision, and clinical utility. Factors that affect discovery and validation should be controlled to overcome pitfalls in biomarker pipeline. Evidence-based biomarker which fulfills regulatory requirements should be introduced into clinical practice by collaboration with research laboratory, diagnostic industry, and clinical laboratory. Rapid development in $\mathrm{CDx}$ would provide a cost-effective best practice for the management of CLD.

\section{Declaration of Conflicting Interests}

The author(s) declared no potential conflicts of interest with respect to the research, authorship, and/or publication of this article.

\section{Funding}

The author(s) received no financial support for the research, authorship, and/or publication of this article.

\section{References}

1. Hong $\mathrm{H}$ and Tong W. Emerging efforts for discovering new biomarkers of liver disease and hepatotoxicity. Biomark Med 2014; 8(2): 143-146.

2. Nallagangula KS, Nagaraj SK, Venkataswamy L, et al. Liver fibrosis: a compilation on the biomarkers status and their significance in disease progress. Future Sci OA 2017; 4(1): FSO250. DOI: 10.4155/fsoa-2017-0083.

3. Gressner OA, Weiskirchen R, and Gressner AM. Evolving concepts of liver fibrogenesis provide new diagnostic and therapeutic options. Comp Hepatol 2007; 6: 7. DOI: 10. 1186/1476-5926-6-7.

4. Ebrahimi H, Naderian M, and Sohrabpour AA. New concepts on pathogenesis and diagnosis of liver fibrosis; a review article. Middle East J Dig Dis 2016; 8(3): 166-178.

5. Scott LF. Hepatic fibrosis. In: Schiff ER, Sorrell MF and Maddrey WC (eds) Schiff's diseases of the liver, 11th ed. Singapore: Wiley-Blackwell, 2012, pp. 297-305.

6. Hunter DJ, Losina E, Guermazi A, et al. A pathway and approach to biomarker validation and qualification for osteoarthritis clinical trials. Curr Drug Targets 2010; 11(5): 536-545.

7. Drucker E and Krapfenbauer K. Pitfalls and limitations in translation from biomarker discovery to clinical utility in predictive and personalized medicine. EPMA J 2013; 4: 7.

8. George P. Bring on the biomarkers. Nature 2011; 469: 156-157.

9. Gangadharan B, Antrobus R, Chittenden D, et al. New approaches for biomarker discovery: the search for liver fibrosis markers in hepatitis C patients. J Proteome Res 2011; 10: 2643-2650.

10. Aebersold R, Anderson L, Caprioli R, et al. Perspective: a program to improve protein biomarker discovery for cancer. J Proteome Res 2005; 4: 1104-1109.

11. Anderson NL. The role of multiple proteome platforms in a pipeline for new diagnostics. Mol Cell Proteomics 2005; 4: 1441-1444.
12. Nies KM, Ivy DD, and Everett DA. The untapped potential of proteomic analysis in pediatric pulmonary hypertension. Proteomics Clin Appl 2014; 8: 862-874.

13. Rifai N, Gillette AM, and Carr AS. Protein biomarker discovery and validation: the long and uncertain path to clinical utility. Nat Biotechnol 2006; 24(8): 971-983.

14. Parker $\mathrm{CE}$ and Borchers $\mathrm{CH}$. Mass spectrometry based biomarker discovery, verification and validation-quality assurance and control of protein biomarker assays. Mol Oncol 2014; 8: 840-858.

15. Farrah T, Deutsch EW, Omenn GS, et al. A high confidence human plasma proteome reference set with estimated concentrations in peptide atlas. Mol Cell Proteomics 2011; 10 : M110.006353.

16. Goossens N, Nakagawa S, Sun X, et al. Cancer biomarker discovery and validation. Trans Cancer Res 2015; 4(3): 256-269.

17. Anderson NL and Anderson NG. The human plasma proteome: history, character, and diagnostic prospects. Mol Cell Proteomics 2002; 1: 845-867.

18. Apple FS, Alan HB, Johanes M, et al. Future biomarkers for detection of ischemia and risk stratification in acute coronary syndrome. Clin Chem 2005; 51(5): 810-824.

19. Paulovich AG, Whiteaker JR, Hoofnagle AN, et al. The interface between biomarker discovery and clinical validation: the tar pit of the protein biomarker pipeline. Proteomics Clin Appl 2008; 2: 1386-1402.

20. Anderson L. Candidate based proteomics in the search for biomarkers of cardiovascular disease. J Physiol 2005; 563(1): 23-60.

21. Fillip S, Vougas K, Zoidakis J, et al. Comparison of depletion strategies for the enrichment of low-abundance proteins in urine. PLoS One 2015; 10(7): e0133773.

22. Steel LF, Trotter MG, Nakajima PB, et al. Efficient and specific removal of albumin from human serum samples. Mol Cell Proteomics 2003; 2(4): 262-270.

23. Xiao Z, Conrads TP, Lucas DA, et al. Direct ampholyte-free liquid-phase isoelectric peptide focusing: application to the human serum proteome. Electrophoresis 2004; 25: 128-133.

24. Hoefkens J. Towards unbiased biomarker discovery. Biomarkers 2010; 1: 19-24.

25. Adkins JN, Susan MV, Auberry KJ, et al. Toward a human blood serum proteome: analysis by multidimensional separation coupled with mass spectrometry. Mol Cell Proteomics 2002; 1(12): 947-955.

26. Tirumalai RS, Chan KC, Prieto DA, et al. Characterization of the low molecular weight human serum proteome. Mol Cell Proteomics 2003; 2(10): 1096-1103.

27. Krastins B, Prakash A, Sarracino DA, et al. Rapid development of sensitive, high-throughput, quantitative and highly selective mass spectrometric targeted immunoassays for clinically important proteins in human plasma and serum. Clin Biochem 2013; 46: 399-410.

28. Marrer E and Dieterle F. Promises of biomarkers in drug development-a reality check. Chem Biol Drug Des 2007; 69: 381-394. 
29. Gillette MA, Mani DR, and Carr SA. Place of pattern in proteomic biomarker discovery. J Proteome Res 2005; 4(4): 1143-1154.

30. Silberring $\mathrm{J}$ and Ciborowski P. Biomarker discovery and clinical proteomics. Trends Analyt Chem 2010; 29(2): 128. DOI: 10.1016/j.trac.2009.11.007.

31. White IR, Patel K, Symonds WT, et al. Serum proteomic analysis focused on fibrosis in patients with hepatitis $\mathrm{C}$ virus infection. J Transl Med 2007; 5: 33. DOI: 10.1186/14795876-5-33.

32. Gangadharan B, Antrobus R, Dwek RA, et al. Novel serum biomarker candidates for liver fibrosis in hepatitis $\mathrm{C}$ patients. Clin Chem 2007; 53(10): 1792-1799.

33. Gangadharan B, Bapat M, Rossa J, et al. Discovery of novel biomarker candidates for liver fibrosis in hepatitis $\mathrm{C}$ patients: a preliminary study. PLoS One 2012: 7(6): e39603. DOI: 10. 1371/journal.pone.0039603.

34. Katrinli S, Ozdil K, Sahin A, et al. Proteomic profiling of HBV infected liver biopsies with different fibrotic stages. Proteome Sci 2017; 15: 7. DOI: 10.1186/s12953-017-0114-4.

35. Vitzthum F, Behrens F, Anderson NL, et al. Proteomics: from basic research to diagnostic application. A review of requirements \& needs. J Proteome Res 2005; 4: 1086-1097.

36. Wild D. The immunoassay handbook. 3rd ed. Amsterdam: Elsevier, 2005.

37. Dybkaer R. Vocabulary for use in measurement procedures and description of reference materials in laboratory medicine. Eur J Clin Chem Biochem 1997; 35: 141-173.

38. Nallagangula KS, Shashidhar KN, Lakshmaiah V, et al. Cirrhosis of liver: interference of serpins in quantification of SERPINA4-a preliminary study. Pract Lab Med 2017; 9: 53-57. DOI: 10.1016/j.plabm.2017.10.002.

39. NCCLS. Evaluation of the linearity of quantitative measurement procedures: a statistical approach, approved guideline. Wayne, PA: NCCLS Document EP6-A, NCCLS, 2003.

40. Lesko LJ and Atkinson AJ. Use of biomarkers and surrogate endpoints in drug development and regulatory decision making: criteria, validation strategies. Annu Rev Pharm Toxicol 2001; 41: 347-366.

41. Bossuyt PM, Reitsma JB, Bruns DE, et al. The STARD statement for reporting studies of diagnostic accuracy: explanation and elaboration. Clin Chem 2003; 49(1): 7-18.

42. Obuchowski NA, Lreber ML, and Wians FH. ROC curves in clinical chemistry: uses, misuses, and possible solutions. Clin Chem 2004; 50: 1118-1125.

43. Sackett DL and Haynes RB. The architecture of diagnostic research. Br Med J 2002; 324: 539-541.

44. Zolg W. The proteomic search for diagnostic biomarkers: lost in translation? Mol Cell Proteomics 2006; 5(10): 1720-1726.

45. Waerner T, Urthaler J, and Krapfenbauer K. The role of laboratory medicine in healthcare: quality requirements of immunoassays, standardization and data management in prospective medicine. EPMA J 2010; 1: 619-626.

46. Sturgeon CM, Hoffman BR, Chan DW, et al. National academy of clinical biochemistry laboratory medicine practice guidelines for use of tumor markers in clinical practice: quality requirements. Clin Chem 2008; 54: e1-e10.

47. Sturgeon C, Hill R, Hortin GL, et al. Taking a new biomarker into routine use-a perspective from the routine clinical biochemistry laboratory. Proteomics Clin Appl 2010; 4: 892-903.

48. Hortin GL, Jortani SA, Ritchie JC, et al. Proteomics: a new diagnostic frontier. Clin Chem 2006; 52: 1218-1222.

49. Ransohoff DF. How to improve reliability and efficiency of research about molecular markers: roles of phases, guidelines, and study design. J Clin Epidemiol 2007; 60: 1205-1219.

50. Hemingway H, Riley RD, and Altman DG. Ten steps towards improving prognosis research. Br Med J 2009; 339: 410-414.

51. Code Federal Regulations, Vol.21 CFR807. http://frwebgate. access.gpo.gov/cgi-bin/get-cfr.cgi?YEAR $=$ current $\&$ TITLE $=21 \&$ PART $=807 \&$ SECTION $=81 \&$ SUBPART $=\&$ TYPE $=$ TEXT (accessed August 2017).

52. Code Federal Regulations, Vol.21 CFR814. http://frwebgate. access.gpo.gov/cgi-bin/get-cfr.cgi?YEAR=current\& TITLE $=21 \& \mathrm{PART}=814 \& \mathrm{SECTION}=1 \& \mathrm{SUBPART}=\&$ TYPE$=$ TEXT (accessed August 2017).

53. Agarwal A, Ressler D, and Snyder G. The current and future state of companion diagnostics. Pharm Personal Med 2015; 8: 99-110. 